\title{
Complex IV subunit 1 defect predicts postoperative survival in hepatocellular carcinoma
}

\author{
PUO-HSIEN LE ${ }^{1}$, SHIH-CHIANG HUANG ${ }^{2}$, SIEW-NA LIM ${ }^{3}$, CHANG-HUA CHOU $^{1}$, \\ TA-SEN YEH ${ }^{4}$, TSE-CHING CHEN ${ }^{2}$, TZUNG-HAI YEN ${ }^{5}$, MING-YAO SU ${ }^{1}$, \\ CHENG-TANG CHIU ${ }^{1}$, CHAU-TING YEH ${ }^{6}$ and WEY-RAN LIN ${ }^{1}$ \\ Departments of ${ }^{1}$ Gastroenterology and Hepatology, ${ }^{2}$ Pathology, ${ }^{3}$ Neurology, ${ }^{4}$ General Surgery and ${ }^{5}$ Nephrology; \\ ${ }^{6}$ Liver Research Center, Linkou Chang Gung Memorial Hospital, Chang Gung University College of Medicine, \\ Taoyuan 333, Taiwan, R.O.C.
}

Received July 24, 2013; Accepted February 20, 2014

DOI: $10.3892 / \mathrm{ol} .2014 .1966$

\begin{abstract}
Mitochondrial oxidative phosphorylation (OXPHOS) is responsible for adenosine triphosphate synthesis and OXPHOS deficiency plays a significant role in tumorigenesis. The defects of mitochondrial-encoded OXPHOS subunits have been found in normal and cirrhotic liver, however their contributions in hepatocellular carcinoma (HCC) are not clear. The present study aimed to examine these defects in resected HCC tissues. In total, 102 human HCC tissues were collected from patients undergoing curative resection, and immunohistochemical staining was performed to assess tissue expression of complex I subunit 6, complex III subunit 3, complex IV subunit 1 (CIV-1) and complex V subunit 6 . Cox proportional hazard model analysis was performed, including all clinicopathological factors, to postoperatively estimate the overall survival rate. The results showed that the majority of HCC tissues contained various degrees of expression defects for OXPHOS subunits. Among these, the major CIV-1 defect (expression defect area of $>25 \%$ of the examined area) $(\mathrm{P}<0.001)$ and early distant metastasis $(\mathrm{P}<0.001)$ were independently associated with the overall survival rate. Kaplan-Meier analysis also demonstrated that the major CIV-1 defect was significantly associated with a poor overall survival rate (log-rank, $\mathrm{P}=0.002$ ). The findings in the present study clearly indicate that the major CIV-1 expression defect may
\end{abstract}

Correspondence to: Dr Wey-Ran Lin, Department of Gastroenterology and Hepatology, Linkou Chang Gung Memorial Hospital, Chang Gung University College of Medicine, 5 Fu-Shin, Taoyuan 333, Taiwan, R.O.C.

E-mail: t12360@adm.cgmh.org.tw

Professor Chau-Ting Yeh, Liver Research Center, Linkou Chang Gung Memorial Hospital, Chang Gung University College of Medicine, 5 Fu-Shin, Taoyuan 333, Taiwan, R.O.C.

E-mail: chautingy@gmail.com

Key words: NADH dehydrogenase, cytochrome $b$, cytochrome $c$ oxidase, ATP synthase serve as an independent negative prognostic factor in $\mathrm{HCC}$ patients following curative resection.

\section{Introduction}

Hepatocellular carcinoma (HCC) is the sixth most common solid cancer and the third leading cause of cancer-related mortality worldwide (1). The occurrence of HCC is multi-factorial and it often develops under an established background of chronic liver diseases (2-4). The most outstanding risk factor in Eastern Asia is chronic hepatitis B virus (HBV) infection, while in Japan, Europe and North America, hepatitis $\mathrm{C}$ virus (HCV) infection is the notable risk factor, synergistically with alcohol abuse (5). Liver resection is potentially a curative therapy in HCC patients who are within the Milan criteria and have an adequate liver reserve. However, tumor recurrence occurs in $>50 \%$ of cases within 5 years following surgery, combining true recurrence of the original cancer, which usually arises within the first 2 years, and de novo tumor formation (6). Early recurrence is associated with microvascular invasion, poor histological differentiation, satellites and multifocal disease, while late recurrence is mainly dependent on the oncogenic potential of underlying chronic liver disease $(7,8)$. Specific molecules have been identified as factors for predicting postoperative survival. These include proline-directed protein kinase FA, mitogen-activated protein kinase phosphatase-1, vascular endothelial growth factor, proliferating cell nuclear antigen, p53, tissue factor, cytokeratin-19, telomerase activity and interleukin-10 (4,9-16). In patients with HBV-related HCC, the HBV basal core promoter mutation and HBV-DNA level in liver tissues also predict a poor postoperative survival rate (17).

Oxidative phosphorylation (OXPHOS) in mitochondria provides biological energy for intracellular metabolic pathways (18). It is particularly significant in hepatocytes as the liver is one of the most energy-consuming organs. In 1930, Warburg proposed that cancer originated from an irreversible injury to mitochondrial OXPHOS, which forced cancer cells to shift to an energy-generation process through glycolysis despite the presence of aerobic conditions. This condition has been named as the Warburg effect (19). It renders cancer cells capable of surviving and proliferating under adverse conditions. 
Mitochondrial DNA (mtDNA) is more susceptible to oxidative damage and has a higher mutation rate than nuclear DNA due to a lack of protective histones, limited DNA repair activities and a proximity to the high rate of reactive oxygen species generated in mitochondria (20-22). As such, the accumulation of mtDNA alterations in cancer cells leading to defects in adenosine triphosphate (ATP) generation through the OXPHOS system is consistent with the theory of the Warburg effect. Mutations in mtDNA have been reported in various types of human cancers (23-25). These findings indicate that defects of the OXPHOS complex with mitochondrial-encoded subunits may be a decisive factor in hepatocarcinogenesis.

The OXPHOS process consists of five complexes, which are complex I (NADH: ubiquinone oxidoreductase), complex II (succinate dehydrogenase), complex III (cytochrome $b c 1$ complex), complex IV (cytochrome $c$ oxidase) and complex V (ATP synthase), and all are localized on the inner mitochondrial membrane. During electron transport, complexes I, III and IV pump protons from the mitochondrial matrix to the inter-membrane space, resulting in an increase in the membrane potential across the inner mitochondrial membrane. Following this, complex $\mathrm{V}$ actively allows the flow of protons back to the matrix, resulting in the generation of energy in the form of ATP from adenosine diphosphate (26). The OXPHOS system consists of 85 subunits as components of various complexes, in which 13 are encoded in mtDNA. These 13 mitochondrial-encoded proteins constitute various subunits that make up four OXPHOS complexes (complex I, III, IV and V) (27). Studies have demonstrated that the mtDNA mutations alter the mitochondrial-encoded subunits and play a significant role in numerous malignancies, including renal oncocytoma, thyroid oncocytic carcinoma, bladder, prostate and colon cancer (28-32).

MtDNA mutations have also been identified in HCC (33-35). These mutations potentially cause the defects of mitochondrial-encoded subunits in the OXPHOS system and result in mitochondrial dysfunction in HCC (36). It has been demonstrated that the defects of complexes III and IV can be detected by immunohistochemical (IHC) staining in normal human and cirrhotic liver during aging. However, these defects were not shown in 27 sections of HCC tissues (37). The actual defects of mitochondrial-encoded subunits in HCC and the association with the outcome remain unclear. The present study aimed to examine the expression of the defects of the mitochondrial-encoded OXPHOS subunits; complex I subunit 6 (CI-6), complex III subunit 3 (CIII-3), complex IV subunit 1 (CIV-1) and complex V subunit 6 (CV-6), in surgically removed HCCs, and their correlations with clinicopathological factors and prognosis.

\section{Patients and methods}

Patients and specimens. Under the approval of the Institutional Review Board, Chang Gung Medical Center (Taoyuan, Taiwan), 102 human HCC tissues with a clear surgical margin were randomly collected from patients undergoing curative resection at Chang Gung Memorial Hospital at Linkou (Taoyuan, Taiwan) between 1996 and 2006 and stored in the Chang Gung tissue bank. The tissues were retrieved from the tissue bank and formalin-fixed and paraffin-embedded for the IHC study. The inclusion criteria included pathological diagnosis of HCC, no anticancer therapy prior to the surgery, curative resection, adequately formalin-fixed and paraffin-embedded tissues, complete clinicopathological data, regular follow-up and reliable medical records. The exclusion criteria included pregnancy, other co-existing malignancies and mortality due to unrelated diseases.

The clinical parameters included age, gender, chronic $\mathrm{HBV}$, chronic HCV, liver cirrhosis, ascites, $\alpha$-fetoprotein (AFP), albumin, bilirubin, prothrombin time, creatinine, aspartate aminotransferase (AST), alanine aminotranferease (ALT), alcohol use (average alcohol consumption of $>210 \mathrm{~g}$ per week in males or $>140$ per week in females over at least a 2-year period), local recurrence, time to local recurrence, distant metastasis, time to distant metastasis, mortality and overall survival time. The pathological findings, including tumor grade, microvascular invasion, macrovascular invasion, capsule, tumor number and largest tumor diameter, were examined by two experienced pathologists without information of the clinical data.

Curative resection was defined as a complete resection of all tumors with the margin free from cancer invasion by histological examination, no tumor thrombus in the main trunk, two major portal branches, hepatic veins or bile duct and no extrahepatic metastasis (38). HCC diagnosis and grading were established according to World Health Organization criteria (39). Macrovascular invasion was defined as the invasion of the tumor into the vessels that can be identified during macroscopic examination or radiological imaging. The definition of microvascular invasion included: i) Presence of tumor cells forming a plug or polyp in a subendothelial location, partially or totally covered by endothelial cells; ii) presence of tumor thrombus, partially or totally covered by the endothelium; iii) vascular structures involved can be portal vein branches, hepatic vein branches or capsule vessels, inside the tumor or closely situated to the tumor edge; and iv) invasion of arteries and lymphatic vessels (40). Local recurrence was defined as intrahepatic recurrence and distant metastasis was equal to extrahepatic metastasis.

Ethics statement. This study was approved by Chang Gung Medical Foundation Institutional Review Board (no. 100-1728B, between 01/08/2011 and 31/07/2014; Taoyuan, Taiwan). The Institutional Review Board waived the requirement for informed consent from the participants as the present study was a retrospectively observational analysis and the information identifying patients was not included in the collected data. Tissue samples were obtained from the Tissue Bank of Linkou Chang Gung Memorial Hospital (Taoyuan, Taiwan) through approval of the committee.

IHC analysis. The IHC stains were performed for detection of the expression defects of CI-6, CIII-3, CIV-1 and CV-6 with rabbit anti-nicotinamide adenine dinucleotide (NADH) dehydrogenase subunit 6 polyclonal antibody (Abcam, Cambridge, MA, USA), goat anti-cytochrome $b$ polyclonal antibody (Santa Cruz Biotechnology, Inc., Santa Cruz, CA, USA), mouse anti-OXPHOS complex IV subunit I monoclonal antibody (Invitrogen Life Technologies, Carlsbad, CA, USA) and rabbit anti-mt-ATP6 polyclonal antibody (Abcam), respectively. Deparaffinized rehydrated sections were treated with $\mathrm{H}_{2} \mathrm{O}_{2}$ 
(3\% in distilled water) for 15 min, pre-incubated with normal serum (goat serum for rabbit anti-NADH dehydrogenase subunit 6 antibody and anti-mt-ATP6 antibody; horse serum for anti-OXPHOS complex IV subunit I monoclonal antibody; and rabbit serum for anti-cytochrome $b$ ) with phosphate-buffered saline in the proportion of 1:10 for $40 \mathrm{~min}$. Following this, the sections were then incubated with the specific primary antibody $\left(1 \mathrm{~h}\right.$ at $37^{\circ} \mathrm{C}$ for anti-NADH dehydrogenase subunit 6 antibody and anti-OXPHOS complex IV subunit I monoclonal antibody; and overnight at $4^{\circ} \mathrm{C}$ for anti-cytochrome $b$ and anti-mt-ATP6 antibody) and the secondary antibody of the VECTASTIN Elite ABC kit [anti-rabbit immunoglobulin G (IgG) for rabbit anti-NADH dehydrogenase subunit 6 antibody and anti-mt-ATP6 antibody; anti-mouse IgG for anti-OXPHOS complex IV subunit I monoclonal antibody; and anti-goat IgG for anti-cytochrome $b$; Vector Laboratories, Inc., Burlingame, CA, USA] for $40 \mathrm{~min}$ at room temperature. Visualization was performed with the 3,3'-diaminobenzidine substrate kit, SK-4100 (Vector Laboratories, Inc.). The stained sections were examined separately by two experienced pathologists, who were blinded to the clinical information. The defect areas (absence of reactivity) in the tumor were estimated and recorded as percentages. If there was a discrepancy in the interpretation, a consensus was reached between the two pathologists by reviewing slides simultaneously. Tissues were classified into two groups based on the percentages of the defect areas in a single cross-section of the HCC samples (major defect, $>25 \%$ HCC area; and minor defect, $\leq 25 \%$ of the $\mathrm{HCC}$ area) for further evaluation.

Statistical analysis. Numerical data are presented as mean \pm standard deviation, while categorical data were expressed as absolute number and percentages. The $\chi^{2}$ test was used for group comparisons involving binary data and independent samples. Mann-Whitney U test was used for the largest tumor size, AFP, AST, ALT, local recurrent time, distal metastatic time and disease-free survival time, due to a skewed distribution. Other numerical data were evaluated by Student's t-test. The results were considered to indicate a statistically significant difference when $\mathrm{P}<0.05$. Univariate and multivariate analyses were performed by Cox proportional hazards regression to identify independent risk factors for mortality. The results were presented with hazard ratio (HR), 95\% confidence interval (CI) and P-value. Survival curves were also analyzed by the Kaplan-Meier curve and log-rank test. All statistical calculations were performed using SPSS, 18.0 software (SPSS, Inc., Chicago, IL, USA).

\section{Results}

Patient characteristics. The age of the patients at the time of surgery ranged between 25 and 89 years, and the male to female ratio was 3.64. The tumor-node-metastasis stage was between stages I and IIIA, according to the American Joint Committee on Cancer Cancer Staging Manual, seventh edition (2010) (41). The cohort of the present study included 51 liver cirrhosis patients, 38 alcohol users, 70 chronic HBV carriers, 17 chronic $\mathrm{HCV}$ carriers and $3 \mathrm{HBV}$ and $\mathrm{HCV}$ co-infection patients. The average follow-up duration was $50.29 \pm 43.50$ months. The rates of mortality, local recurrence, distal metastasis, 5-year survival and 5-year disease-free survival were 36.27, 64.71, $39.22,37.25$ and $22.55 \%$, respectively.

Clinicopathological characteristics. The majority of the HCC tissues contained various degrees of mitochondrial-encoded OXPHOS enzyme defects. The expression defects of CI-6, CIII-3, CIV-1 and CV-6 were present in 100, 98, 98 and $93 \%$ of the tissues assessed, respectively. The major and minor defects were defined as IHC staining defect area of $>25 \%$ and $\leq 25 \%$, respectively (Fig. 1). According to this definition, the frequency of the major defect for CI-6, CIV-1, CIII-3 and CV-6 was 67.7, $50.0,31.3$ and $24.5 \%$, respectively. The CI-6 major-defect group had lower ALT levels compared with the minor-defect group [35 (10-280) and 59 (9-371) U/l, P=0.038]. The CIII-3 major-defect group had fewer encapsulated tumors (56.25 and $75.71 \%, \mathrm{P}=0.047)$ and higher albumin levels $(4.03 \pm 0.44$ and $3.67 \pm 0.68 \mathrm{~g} / \mathrm{dl}, \mathrm{P}=0.002)$, compared with the minor-defect group. The CIV-1 major-defect group had higher AFP levels [204.08 (0.9-89637.7) and 21.92 (2-286980) ng/ml, P=0.021] and higher AST levels [63 (12-351) and $36(11-278) \mathrm{U} / 1, \mathrm{P}=0.018$ ] compared with the minor-defect group. The CV-6 major-defect group was older $(63.12 \pm 9.80$ and $53.86 \pm 15.53$ years, $\mathrm{P}=0.001)$ compared with the minor-defect group. There was no other significant difference in clinicopathological parameters between the major- and minor-defect groups (Table I).

Clinicopathological parameters and OXPHOS expression defects associated with post-operative survival in HCC. Univariate analysis revealed that ascites (HR, 6.016; 95\% CI, 2.364-15.309; $\mathrm{P}<0.001$ ), albumin levels (HR, 0.524; $95 \%$ CI, 0.304-0.905; $\mathrm{P}=0.020$ ), time to local recurrence (HR, 0.948; 95\% CI, 0.927-0.970, $\mathrm{P}<0.001)$, time to distant metastasis (HR, 0.934; 95\% CI, 0.915-0.953; $\mathrm{P}<0.001$ ), disease-free survival time (HR, 0.948; 95\% CI, 0.927-0.969; P<0.001) and major CIV-1 defect (HR, 3.050; 95\% CI, 1.471-6.324; $\mathrm{P}=0.003$ ) were associated with the overall survival time (Table II). These six factors were further analyzed by multivariate analysis. It was found that the major CIV-1 defect (HR, 5.676; 95\% CI, 2.243-14.360; $\mathrm{P}<0.001)$ and the time to distant metastasis (HR, 0.924; 95\% CI, 0.894-0.955; $\mathrm{P}<0.001)$ were significant independent predictive factors for overall survival time (Table III). Kaplan-Meier survival analysis using the log-rank test (Fig. 2) also showed that the CIV-1 major-defect group had an unfavorable survival time compared with that of the minor-defect group ( $\mathrm{P}=0.002)$. Additionally, the major CI-6 and CV-6 defect groups appeared to have a poor survival rate within 8 years, but it did not reach statistical significance. There was no significant difference between major- and minor-defect groups of CIII-3, according to the Kaplan-Meier survival curve.

\section{Discussion}

According to the present study, the majority of the HCC tissues contained various degrees of mitochondrial-encoded OXPHOS complex defects, indicating the impairment of ATP production by pyruvate oxidation in mitochondria. This result is compatible with the phenomenon of the Warburg effect, indicating that energy is mainly supplied by glycolysis in cancer cells (19). To understand whether the degree of OXPHOS defects correlate 

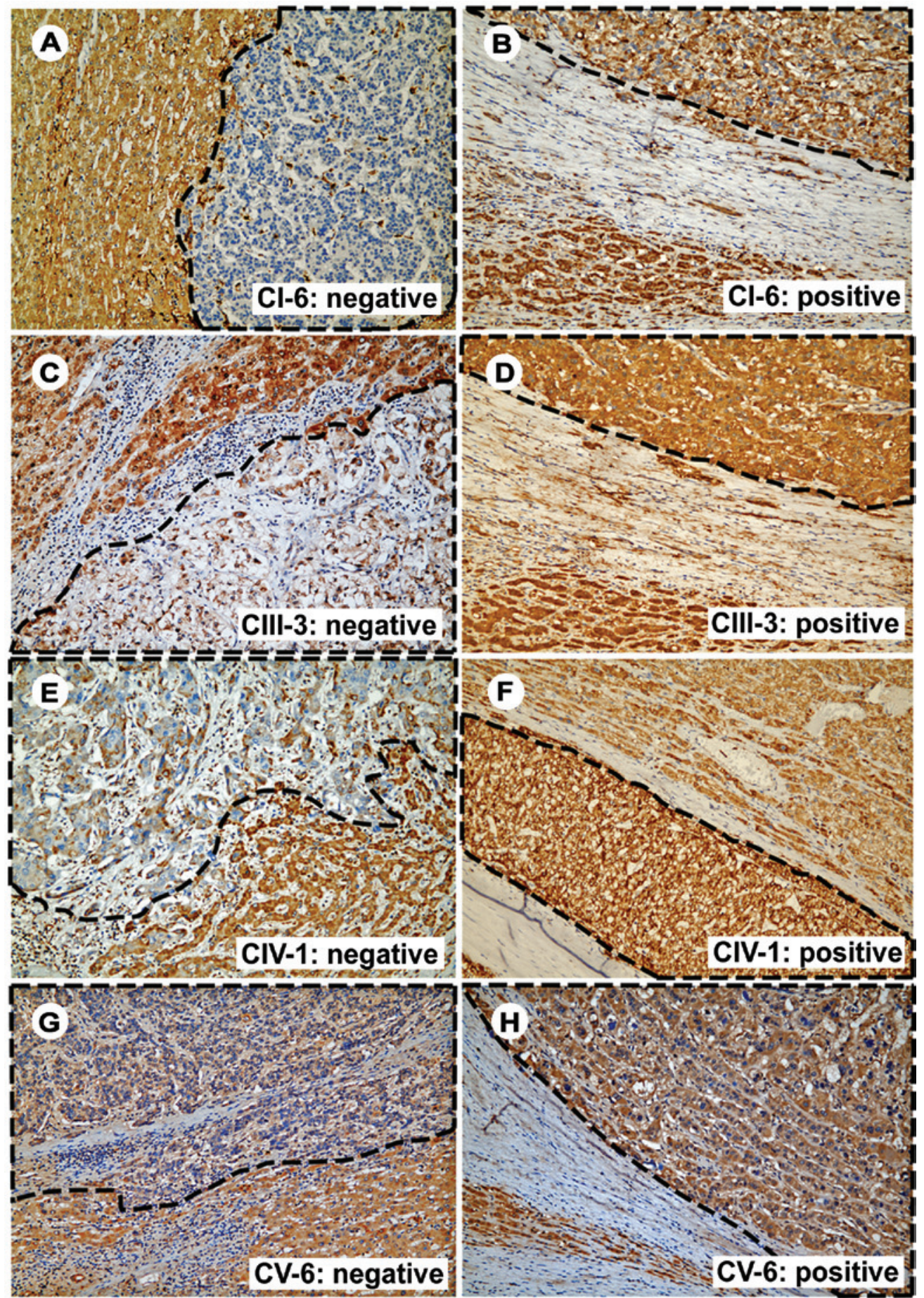

Figure 1. Immunohistochemical staining of (A and B) complex I subunit 6, (C and D) complex III subunit 3, (E and F) complex IV subunit 1 and (G and H) complex V subunit 6 in hepatocellular carcinoma, within the dotted line.

with clinicopathological presentation and prognosis, the major and minor defects were defined as IHC staining defect area of $>25 \%$ (major) and $\leq 25 \%$ (minor). The frequencies of the major defect were as follows: CI-6, 67.65\%; CIV-1, 50.00\%; CIII-3, 31.37\%; and CV-6, 24.51\%). These results indicate that HCC tissues tend to have a larger portion of CI-6 and CIV-1 defects, rather than CIII-3 and CV-6. It has been indicated that the OXPHOS enzyme defects are associated with aging (37). However, in the present study, only the major CV-6 defect was correlated with age $(63.12 \pm 9.80$ and $53.86 \pm 15.53$ years, $\mathrm{P}=0.001$ ), indicating that other mechanisms are responsible for the generation of these defects in HCC, rather than aging. Through univariate and multivariate analyses, the major CIV-1 defect was found to be a negative predictive factor for HCC patients following curative resection.

Complex IV is the terminal oxidase of the respiratory chain in the mitochondria. In mammals, it contains 13 subunits, of which 3 catalytic subunits (subunit 1, 2 and 3) are encoded by the mitochondrial genes. The remaining 10 subunits are encoded by nuclear DNA and are suspected to be involved in the regulation and/or assembly of the complex (41). Complex IV represents the rate-limiting enzyme 


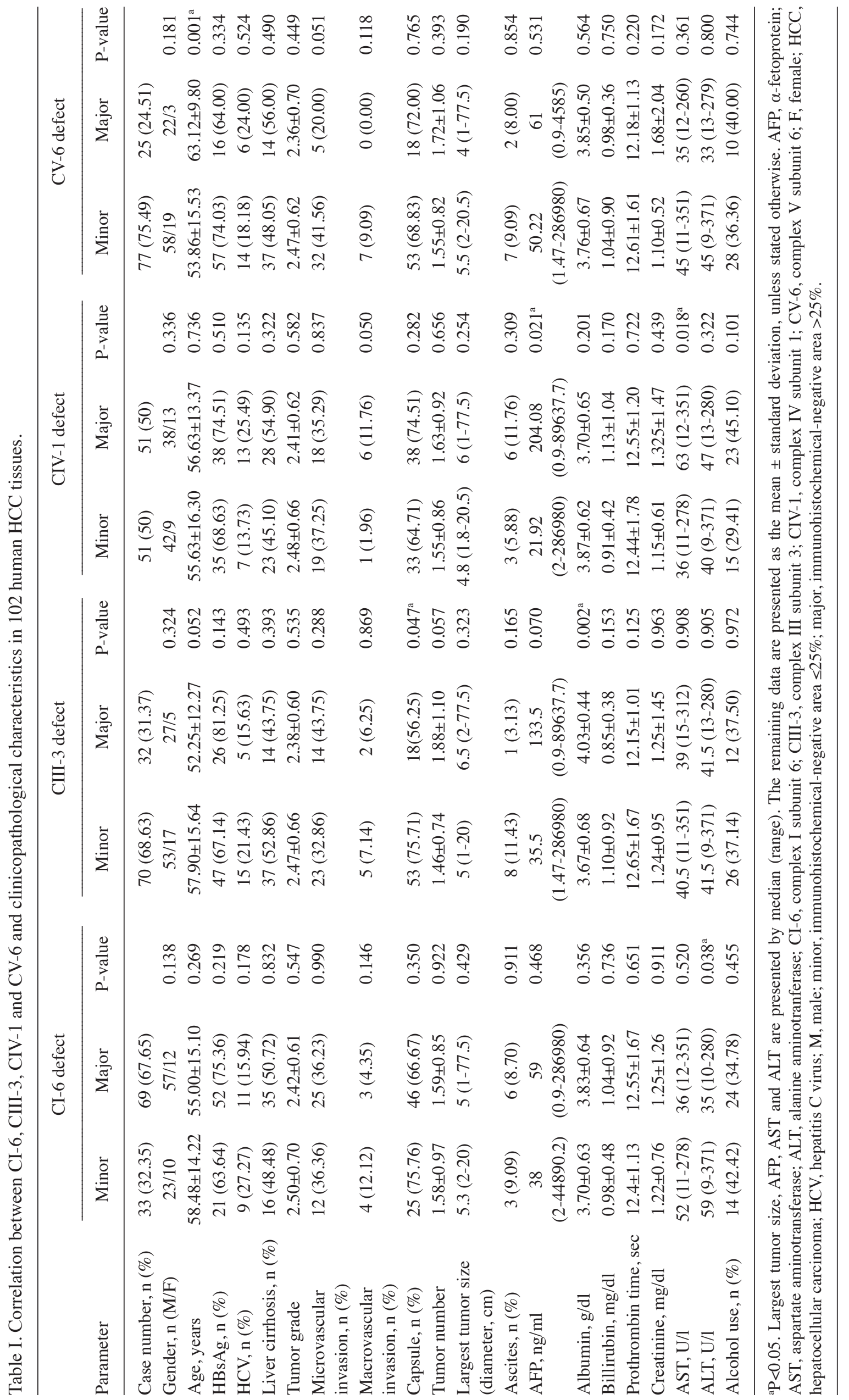


Table II. Univariate analysis of parameters associated with overall survival time.

\begin{tabular}{|c|c|c|c|c|c|}
\hline Parameter & Patient data & SE & HR & $95 \% \mathrm{CI}$ & P-value \\
\hline Gender, male & $80(78.4 \%)^{\mathrm{a}}$ & 0.384 & 0.964 & $0.454 \sim 2.046$ & 0.924 \\
\hline Age, years & $56.13 \pm 14.84$ & 0.011 & 1.012 & $0.990-1.034$ & 0.292 \\
\hline HBsAg & $73(71.6 \%)^{\mathrm{a}}$ & 0.391 & 0.999 & $0.464 \sim 2.150$ & 0.999 \\
\hline $\mathrm{HCV}$ & $20(19.6 \%)^{\mathrm{a}}$ & 0.485 & 0.727 & $0.281 \sim 1.879$ & 0.511 \\
\hline Liver cirrhosis & $51(50.0 \%)^{\mathrm{a}}$ & 0.332 & 0.919 & $0.479 \sim 1.762$ & 0.798 \\
\hline Tumor grade & $2.44 \pm 0.64$ & 0.255 & 1.001 & $0.608-1.649$ & 0.996 \\
\hline Microvascular invasion & $37(36.3 \%)^{\mathrm{a}}$ & 0.366 & 1.181 & $0.577 \sim 2.418$ & 0.649 \\
\hline Macrovascular invasion & $7(6.9 \%)^{\mathrm{a}}$ & 0.535 & 1.799 & $0.630 \sim 5.139$ & 0.273 \\
\hline Capsule & $71(69.6 \%)^{\mathrm{a}}$ & 0.386 & 1.272 & $0.597 \sim 2.709$ & 0.533 \\
\hline Tumor number & $1.59 \pm 0.88$ & 0.181 & 1.136 & $0.797-1.619$ & 0.481 \\
\hline Largest tumor size (diameter, cm) & $5.1(1.0-77.5)^{\mathrm{b}}$ & 0.012 & 1.012 & $0.989-1.037$ & 0.313 \\
\hline Ascites & $9(8.8 \%)^{\mathrm{a}}$ & 0.477 & 6.016 & $2.364 \sim 15.309$ & $<0.001^{\mathrm{c}}$ \\
\hline $\mathrm{AFP}(\mathrm{ng} / \mathrm{ml})$ & $50.26(0.9-286980)^{\mathrm{b}}$ & 0.000 & 1.000 & $1.000 \sim 1.000$ & 0.585 \\
\hline Albumin (g/dl) & $3.78 \pm 0.64$ & 0.278 & 0.524 & $0.304-0.905$ & $0.020^{\mathrm{c}}$ \\
\hline Billirubin (mg/d) & $1.02 \pm 0.80$ & 0.219 & 1.163 & $0.758-1.785$ & 0.490 \\
\hline Prothrombin time (sec) & $12.50 \pm 1.51$ & 0.083 & 1.159 & $0.985-1.365$ & 0.076 \\
\hline Creatinine $(\mathrm{mg} / \mathrm{dl})$ & $1.24 \pm 1.12$ & 0.111 & 1.171 & $0.942-1.456$ & 0.155 \\
\hline $\operatorname{AST}(\mathrm{U} / \mathrm{l})$ & $39.5(11-351)^{\mathrm{b}}$ & 0.002 & 1.004 & $1.000-1.009$ & 0.073 \\
\hline $\operatorname{ALT}(\mathrm{U} / \mathrm{l})$ & $41.5(9-371)^{b}$ & 0.003 & 0.999 & $0.993 \sim 1.005$ & 0.692 \\
\hline Alcohol use & $38(37.3 \%)^{\mathrm{a}}$ & 0.332 & 1.387 & $0.723 \sim 2.660$ & 0.325 \\
\hline Local recurrent time (month) & $14(0.07-194)^{\mathrm{b}}$ & 0.011 & 0.948 & $0.927-0.970$ & $<0.001^{\mathrm{c}}$ \\
\hline Distal metastatic time (month) & $34.5(0.07-194)^{\mathrm{b}}$ & 0.010 & 0.934 & $0.915-0.953$ & $<0.001^{\mathrm{c}}$ \\
\hline Disease free survival time (month) & $14(0.07-194)^{\mathrm{b}}$ & 0.011 & 0.948 & $0.927-0.969$ & $<0.001^{\mathrm{c}}$ \\
\hline Major complex I subunit 6 defect & $69(67.6 \%)^{\mathrm{a}}$ & 0.371 & 1.326 & $0.641-2.742$ & 0.446 \\
\hline Major complex III subunit 3 defect & $32(31.4 \%)^{\mathrm{a}}$ & 0.352 & 0.950 & $0.477-1.893$ & 0.885 \\
\hline Major complex IV subunit 1 defect & $51(50.0 \%)^{\mathrm{a}}$ & 0.372 & 3.050 & $1.471 \sim 6.324$ & $0.003^{\mathrm{c}}$ \\
\hline Major complex V subunit 6 defect & $25(24.5 \%)^{\mathrm{a}}$ & 0.361 & 1.255 & $0.618-2.546$ & 0.530 \\
\hline
\end{tabular}

${ }^{\mathrm{a} N u m b e r}(\%)$; ${ }^{\mathrm{b}}$ median (range); ${ }^{\mathrm{c}} \mathrm{P}<0.05$. Data are presented as the mean \pm standard deviation, unless stated otherwise. SE, standard error; HR, hazard ratio; CI, confidence interval; AFP, $\alpha$-fetoprotein; AST, aspartate aminotransferase; ALT, alanine aminotranferase; HCV, hepatitis C virus; major, immunohistochemical-negative area $>25 \%$.

Table III. Multivariate analysis of parameters associated with overall survival time.

\begin{tabular}{|c|c|c|c|c|}
\hline Parameter & SE & HR & $95 \% \mathrm{CI}$ & P-value \\
\hline Ascites & 0.500 & 1.697 & $0.637 \sim 4.521$ & 0.290 \\
\hline Albumin, g/dl & 0.341 & 1.012 & $0.519 \sim 1.975$ & 0.971 \\
\hline Local recurrent time, month & 0.607 & 0.791 & $0.241 \sim 2.598$ & 0.699 \\
\hline Distal metastatic time, month & 0.017 & 0.924 & $0.894 \sim 0.955$ & $<0.001^{\mathrm{a}}$ \\
\hline Disease-free survival time, month & 0.609 & 1.262 & $0.383 \sim 4.159$ & 0.702 \\
\hline Major complex IV subunit 1 defect & 0.474 & 5.676 & $2.243 \sim 14.360$ & $<0.001^{\mathrm{a}}$ \\
\hline
\end{tabular}

${ }^{a} \mathrm{P}<0.05$. SE, standard error; HR, hazard ratio; CI, confidence interval; major, immunohistochemical-negative area $>25 \%$.

of the mitochondrial respiratory chain and its activity is an indicator of the oxidative capacity of the cells. It is therefore fated to be a central site of regulation of oxidative phosphorylation, proton pumping efficiency, ATP and reactive oxygen species production, which in turn affect cell signaling and survival $(42,43)$. Complex IV can also be incorporated into larger structures containing complex I, II and III, and the mobile electron carriers, cytochrome $c$ and ubiquinol, to form functional supercomplexes; respirasomes $(44,45)$. These supercomplexes may stabilize the individual complexes (46) to enhance respiration due to coordinated channeling of electrons (47). Due to the significance of complex IV, organisms 
$\mathbf{A}$

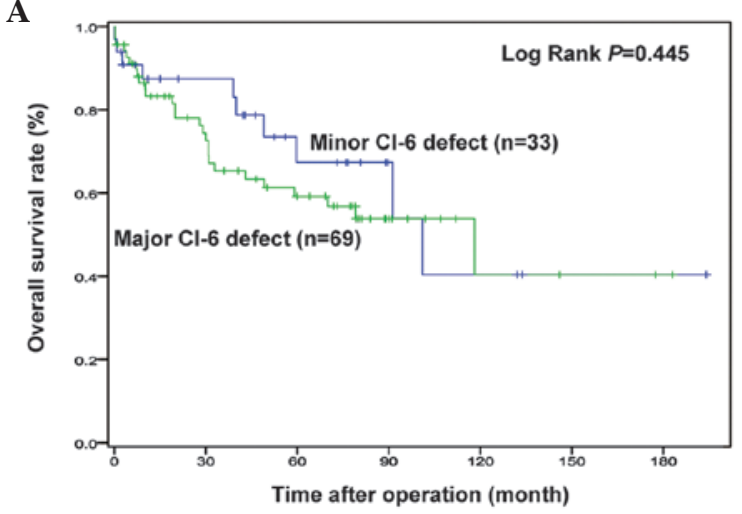

C

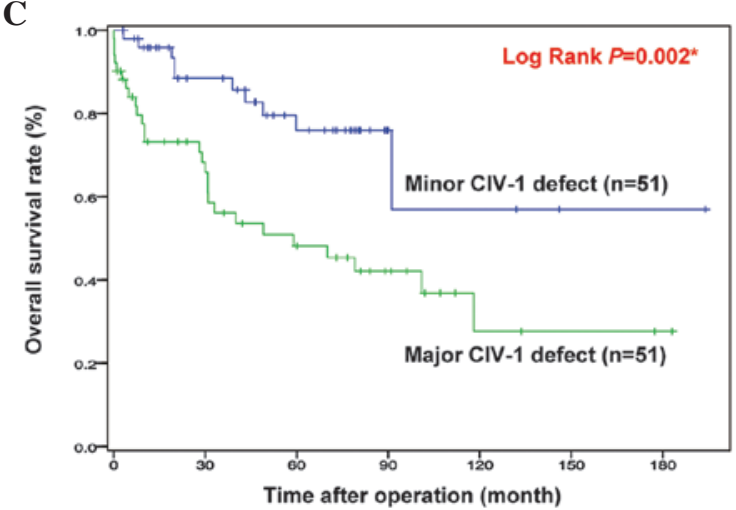

B

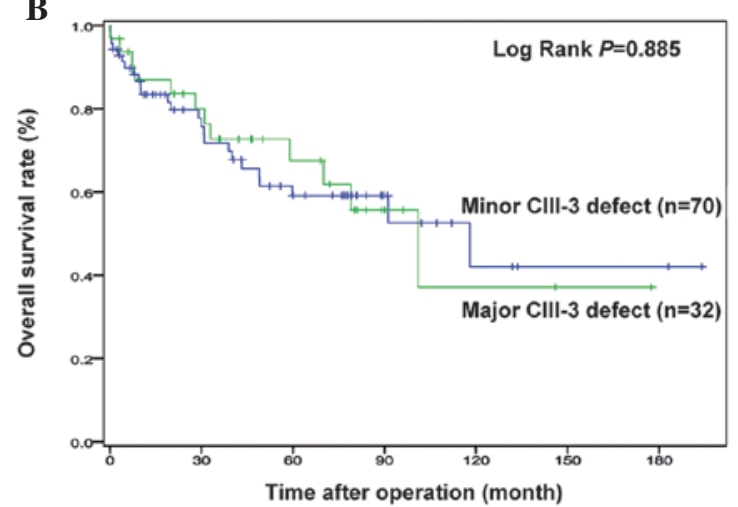

D

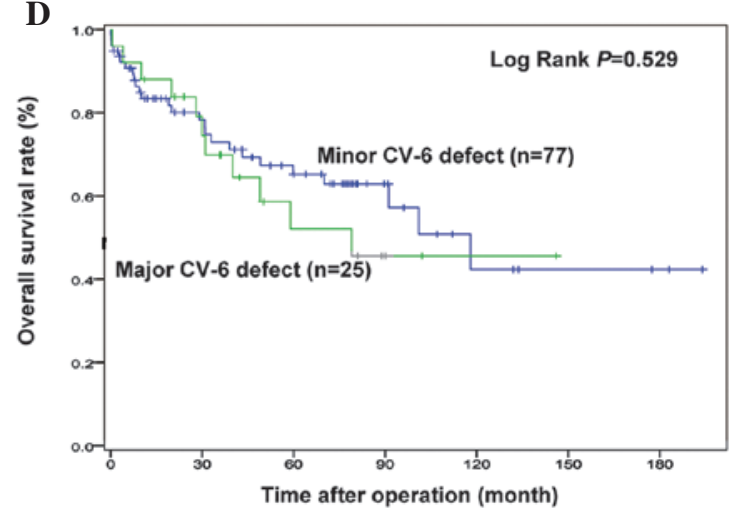

Figure 2. Overall survival curves of hepatocellular carcinoma with (A) complex I subunit 6 (CI-6), (B) complex III subunit 3 (CIII-3), (C) complex IV subunit 1 (CIV-1) and (D) complex V subunit 6 (CV-6) defects, assessed by Kaplan-Meier survival estimates and the log-rank test.

have evolved various levels of regulation for its activity. A defect of complex IV has been proved to result in numerous diseases, including Leber hereditary optic neuropathy, Leigh syndrome, recurrent myoglobinuria mitochondrial disorder, deafness sensorineural mitochondrial disorder and colorectal cancer (48-54). However, their roles in HCC are not clear. The present study firstly revealed the potential significance of the CIV-I defects in HCC. Further experiments involving knocking out the CIV-I gene by small hairpin RNA in the Huh7 HCC cell line will be performed to demonstrate their effects on liver tumor.

It has been demonstrated that OXPHOS protein defects can be found in normal and cirrhotic human liver. A study by Müller-Höcker et al (37) enrolled 107 normal livers (including $11 \mathrm{HCC}$ cases) and 64 cirrhotic livers (including 16 HCC cases) and aimed to detect the respiratory chain protein (complex II, III and IV) and complex V defects in normal and cirrhotic liver during aging. Enzyme histochemistry was performed to detect complex II, IV and V, and immunohistochemistry with polyclonal antibody (against both nuclear and mitochondrial subunits) was conducted for detection of total complex III and complex IV subunits 2, 3 and 4. In normal livers, the respiratory chain defects were detected in $57 \%$ cases, and $87 \%$ in advanced age ( $>50$ years old). In cirrhotic liver, the overall frequency of defects was higher (78\%) compared with normal liver, but was inverse $(60 \%)$ in advanced age. However, there were no defects of complex II, III, IV and V observed in $27 \mathrm{HCC}$ tissues in their study. On the contrary, the present study showed that the majority of resected HCC tissues contain various degrees of mitochondrial-encoded subunits defects. The conflicting results may be due to the varied HCC tissue origins (metastatic and primary), various OXPHOS enzymes examined and different methods performed. Nevertheless, the results of the present study firstly demonstrated that the majority of HCCs contain mitochondrial-encoded subunit defects, indicating a potential role of these defects in hepatocarcinogenesis.

MtDNA mutations have been detected in HCC. A study by Lee et al (33) examined the D-loop mutations and mtDNA numbers in $61 \mathrm{HCCs}$ and the corresponding non-tumor sections. The results showed that $39.3 \%$ of HCCs carried somatic mutations in the D-loop of mtDNA. A significant decrease in the copy number of mtDNA was also detected in $60.5 \%$ of patients with HCC (33). A small-scale study examining $18 \mathrm{HCC}$ patients also demonstrated a significant decrease of mtDNA copy number, particularly in females but not in males with HCC (34). Another study examined $44 \mathrm{HCCs}$ and in total 13 somatic mtDNA mutations were found in 11 HCC samples (35). Among these mutations, the T6768C (CIV-1), G7976A (complex IV subunit II), G9267 (complex IV subunit 3) and A111708 (complex I subunit 4) mutations could result in amino acid substitutions in the highly conserved regions and have the potential to cause mitochondrial dysfunction in HCC. Although the mutation of mtDNA was not examined in the present study, the mitochondrial-encoded OXPHOS subunit defects shown may have at least partially resulted from mtDNA mutations and the decrease of mtDNA copy number.

There were limitations in the present study regarding the case number, the choice of specimens and the lack of western blotting, reverse transcription polymerase chain reaction and mtDNA 
analysis. In total, $102 \mathrm{HCC}$ tissues were collected with an average follow-up duration of 50.29 months (range, 0.07-194 months). The case number appeared to not be enough for subgroup analyses. Due to the strength of the IHC staining, it was more difficult to evaluate objectively and it was not associated with prognosis (data not shown); the percentages of IHC stain defect areas were chosen in a single cross-section of HCC without significant necrosis to evaluate the degree of the OXPHOS complex defects. The same scoring system read by the pathologists is also widely used in clinical practice, including HER2 in breast cancer and Ki-67 in neuroendocrine tumor $(55,56)$. Although it was easy for clinical applications, it may have bias between different pathologists when scoring the defects areas. We hypothesize that this bias can be reduced by repeated reading and experienced examiners.

To the best of our knowledge, the present study was the first to demonstrate the correlation between OXPHOS subunit defects and overall survival time in HCC patients. The results showed that the majority of HCCs contained mitochondrial-encoded OXPHOS subunit defects. Among these, the major CIV-1 defect was a negative predictor for post-operative survival in HCC. It may provide a simple way to predict the outcome in this group of patients.

\section{Acknowledgements}

This study was supported by the grants from the National Science Council (NSC100-2314-B-182A-056) and Chang Gung Medical Research Council (CMRPG3A0921), Taiwan, Republic of China.

\section{References}

1. Forner A, Llovet JM and Bruix J: Hepatocellular carcinoma. Lancet 379: 1245-1255, 2012.

2. Sherman M: Hepatocellular carcinoma: epidemiology, surveillance, and diagnosis. Semin Liver Dis 30: 3-16, 2010.

3. Marra M, Sordelli IM, Lombardi A, et al: Molecular targets and oxidative stress biomarkers in hepatocellular carcinoma: an overview. J Transl Med 9: 171, 2011.

4. Shen HM and Ong CN: Mutations of the p53 tumor suppressor gene and ras oncogenes in aflatoxin hepatocarcinogenesis. Mut Res 366: 23-44, 1996.

5. El-Serag HB: Hepatocellular carcinoma. N Engl J Med 365: 1118-1127, 2011.

6. Llovet JM, Schwartz M and Mazzaferro V: Resection and liver transplantation for hepatocellular carcinoma. Semin Liver Dis 25: 181-200, 2005.

7. Hoshida Y, Villanueva A, Kobayashi M, et al: Gene expression in fixed tissues and outcome in hepatocellular carcinoma. N Engl J Med 359: 1995-2004, 2008.

8. Imamura H, Matsuyama Y, Tanaka E, et al: Risk factors contributing to early and late phase intrahepatic recurrence of hepatocellular carcinoma after hepatectomy. J Hepatol 38: 200-207, 2003.

9. Chau GY, Wu CW, Lui WY, et al: Serum interleukin-10 but not interleukin- 6 is related to clinical outcome in patients with resectable hepatocellular carcinoma. Ann Surg 231: 552-558, 2000

10. Hsu YC, Fu HH, Jeng YM, Lee PH and Yang SD: Proline-directed protein kinase FA is a powerful and independent prognostic predictor for progression and patient survival of hepatocellular carcinoma. J Clin Oncol 24: 3780-3788, 2006.

11. Kitamoto M, Nakanishi T, Kira S, et al: The assessment of proliferating cell nuclear antigen immunohistochemical staining in small hepatocellular carcinoma and its relationship to histologic characteristics and prognosis. Cancer 72: 1859-1865, 1993.

12. Kobayashi T, Kubota K, Takayama T and Makuuchi M: Telomerase activity as a predictive marker for recurrence of hepatocellular carcinoma after hepatectomy. Am J Surg 181: 284-288, 2001
13. Poon RT, Lau CP, Ho JW, Yu WC, Fan ST and Wong J: Tissue factor expression correlates with tumor angiogenesis and invasiveness in human hepatocellular carcinoma. Clin Cancer Res 9: 5339-5345, 2003.

14. Poon RT, Ng IO, Lau C, et al: Serum vascular endothelial growth factor predicts venous invasion in hepatocellular carcinoma: a prospective study. Ann Surg 233: 227-235, 2001.

15. Tsujita E, Taketomi A, Gion T, et al: Suppressed MKP-1 is an independent predictor of outcome in patients with hepatocellular carcinoma. Oncology 69: 342-347, 2005.

16. Uenishi T, Kubo S, Yamamoto T, et al: Cytokeratin 19 expression in hepatocellular carcinoma predicts early postoperative recurrence. Cancer Sci 94: 851-857, 2003.

17. Yeh CT, So M, Ng J, et al: Hepatitis B virus-DNA level and basal core promoter A1762T/G1764A mutation in liver tissue independently predict postoperative survival in hepatocellular carcinoma. Hepatology 52: 1922-1933, 2010.

18. Schapira AH: Mitochondrial disease. Lancet 368: 70-82, 2006.

19. Warburg O: The Metabolism of Tumors. Arnold Constable, London, pp 254-270, 1930.

20. Maynard S, Schurman SH, Harboe C, de Souza-Pinto NC and Bohr VA: Base excision repair of oxidative DNA damage and association with cancer and aging. Carcinogenesis 30: 2-10, 2009.

21. Croteau DL and Bohr VA: Repair of oxidative damage to nuclear and mitochondrial DNA in mammalian cells. J Biol Chem 272: 25409-25412, 1997.

22. Wallace DC: A mitochondrial paradigm of metabolic and degenerative diseases, aging, and cancer: a dawn for evolutionary medicine. Annu Rev Genet 39: 359-407, 2005.

23. Brandon M, Baldi P and Wallace DC: Mitochondrial mutations in cancer. Oncogene 25: 4647-4662, 2006.

24. Chatterjee A, Mambo E and Sidransky D: Mitochondrial DNA mutations in human cancer. Oncogene 25: 4663-4674, 2006.

25. Lee HC and Wei YH: Mitochondrial DNA instability and metabolic shift in human cancers. Int J Mol Sci 10: 674-701, 2009.

26. Poyton RO and McEwen JE: Crosstalk between nuclear and mitochondrial genomes. Annu Rev Biochem 65: 563-607, 1996.

27. Chandra D and Singh KK: Genetic insights into OXPHOS defect and its role in cancer. Biochim Biophys Acta 1807: 620-625, 2011.

28. Mayr JA, Meierhofer D, Zimmermann F, et al: Loss of complex I due to mitochondrial DNA mutations in renal oncocytoma. Clin Cancer Res 14: 2270-2275, 2008.

29. Dasgupta S, Hoque MO, Upadhyay S and Sidransky D: Mitochondrial cytochrome B gene mutation promotes tumor growth in bladder cancer. Cancer Res 68: 700-706, 2008.

30. Bonora E, Porcelli AM, Gasparre G, et al: Defective oxidative phosphorylation in thyroid oncocytic carcinoma is associated with pathogenic mitochondrial DNA mutations affecting complexes I and III. Cancer Res 66: 6087-6096, 2006.

31. Namslauer I, Dietz MS and Brzezinski P: Functional effects of mutations in cytochrome c oxidase related to prostate cancer. Biochim Biophys Acta 1807: 1336-1341, 2011.

32. Bernstein C, Facista A, Nguyen H, et al: Cancer and age related colonic crypt deficiencies in cytochrome c oxidase I. World J Gastrointest Oncol 2: 429-442, 2010.

33. Lee HC, Li SH, Lin JC, Wu CC, Yeh DC and Wei YH: Somatic mutations in the D-loop and decrease in the copy number of mitochondrial DNA in human hepatocellular carcinoma. Mutat Res 547: 71-78, 2004.

34. Yin PH, Lee HC, Chau GY, et al: Alteration of the copy number and deletion of mitochondrial DNA in human hepatocellular carcinoma. Br J Cancer 90: 2390-2396, 2004.

35. Yin PH, Wu CC, Lin JC, Chi CW, Wei YH and Lee HC: Somatic mutations of mitochondrial genome in hepatocellular carcinoma. Mitochondrion 10: 174-182, 2010.

36. Greaves LC, Reeve AK, Taylor RW and Turnbull DM: Mitochondrial DNA and disease. J Pathol 226: 274-286, 2012.

37. Müller-Höcker J, Aust D, Rohrbach H, et al: Defects of the respiratory chain in the normal human liver and in cirrhosis during aging. Hepatology 26: 709-719, 1997.

38. Shah SA, Cleary SP, Wei AC, et al: Recurrence after liver resection for hepatocellular carcinoma: risk factors, treatment, and outcomes. Surgery 141: 330-339, 2007.

39. Theise ND, Curado MP, Franceschi S, et al: WHO classification of tumours of the digestive system. In: Hepatocellular Carcinoma. Bosman FT, Carneiro F, Hruban RH and Theise ND (eds). 4th edition. IARC Press, Lyon, pp 205-216, 2010.

40. Rodríguez-Perálvarez M, Luong TV, Andreana L, Meyer T, Dhillon AP and Burroughs AK: A systematic review of microvascular invasion in hepatocellular carcinoma: diagnostic and prognostic variability. Ann Surg Oncol 20: 325-339, 2013. 
41. Edge SE, Byrd DR, Compton CC, et al (eds.): AJCC Cancer Staging Manual and Handbook. 7th ed. Springer, New York, NY, USA, 2010.

42. Srinivasan S and Avadhani NG: Cytochrome c oxidase dysfunction in oxidative stress. Free Radic Biol Med 53: 1252-1263, 2012.

43. Arnold S: The power of life - cytochrome c oxidase takes center stage in metabolic control, cell signalling and survival. Mitochondrion 12: 46-56, 2012.

44. Schägger $\mathrm{H}$ and Pfeiffer K: The ratio of oxidative phosphorylation complexes I-V in bovine heart mitochondria and the composition of respiratory chain supercomplexes. J Biol Chem 276 : 37861-37867, 2001.

45. Acín-Pérez R, Fernández-Silva P, Peleato ML, Pérez-Martos A and Enriquez JA: Respiratory active mitochondrial supercomplexes. Mol Cell 32: 529-539, 2008.

46. Acín-Pérez R, Bayona-Bafaluy MP, Fernández-Silva $\mathrm{P}$, et al: Respiratory complex III is required to maintain complex I in mammalian mitochondria. Mol Cell 13: 805-815, 2004.

47. Schäfer E, Seelert H, Reifschneider NH, Krause F, Dencher NA and Vonck J: Architecture of active mammalian respiratory chain supercomplexes. J Biol Chem 281: 15370-15375, 2006.

48. Brown MD, Yang CC, Trounce I, Torroni A, Lott MT and Wallace DC: A mitochondrial DNA variant, identified in Leber hereditary optic neuropathy patients, which extends the amino acid sequence of cytochrome c oxidase subunit I. Am J Hum Genet 51: 378-385, 1992

49. Varlamov DA, Kudin AP, Vielhaber S, et al: Metabolic consequences of a novel missense mutation of the mtDNA CO I gene. Hum Mol Genet 11: 1797-1805, 2002.
50. Lucioli S, Hoffmeier K, Carrozzo R, Tessa A, Ludwig B and Santorelli FM: Introducing a novel human mtDNA mutation into the Paracoccus denitrificans COX I gene explains functional deficits in a patient. Neurogenetics 7: 51-57, 2006.

51. Karadimas CL, Greenstein P, Sue CM, et al: Recurrent myoglobinuria due to a nonsense mutation in the COX I gene of mitochondrial DNA. Neurology 55: 644-649, 2000.

52. Pandya A, Xia XJ, Erdenetungalag R, et al: Heterogenous point mutations in the mitochondrial tRNA Ser(UCN) precursor coexisting with the A1555G mutation in deaf students from Mongolia. Am J Hum Genet 65: 1803-1806, 1999.

53. Greaves LC, Preston SL, Tadrous PJ, et al: Mitochondrial DNA mutations are established in human colonic stem cells, and mutated clones expand by crypt fission. Proc Natl Acad Sci USA 103: 714-719, 2006.

54. Namslauer I and Brzezinski P: A mitochondrial DNA mutation linked to colon cancer results in proton leaks in cytochrome c oxidase. Proc Natl Acad Sci USA 106: 3402-3407, 2009.

55. Wolff AC, Hammond ME, Hicks DG, et al: Recommendations for human epidermal growth factor receptor 2 testing in breast cancer: American Society of Clinical Oncology/College of American Pathologists clinical practice guideline update. J Clin Oncol 31: 3997-4013, 2013

56. Rindi G, Arnold R, Bosman FT, et al: Nomenclature and classification of neuroendocrine neoplasms of the digestive system. In: WHO Classification of Tumours of the Digestive System. Bosman TF, Carneiro F, Hruban RH, Theise ND (eds). 4th ed. International Agency for Research on Cancer (IARC), Lyon, France, pp13, 2010. 